between imipramine and placebo in their effects on depressed mood-and depressed mood is, I suppose, the most important single manifestation of a depressive illness.-I am, etc.,

$$
\begin{aligned}
& \text { The Bethlem Royal Hospital, E. H. HARE. } \\
& \text { Beckenham, Kent. }
\end{aligned}
$$

\title{
Services for the Elderly
}

SIR,-Dr. R. Ll. Meyrick (3 April, p. 925) continues to press his argument that geriatric units are unnecessary. True enough, on the figures presented in the King Edward's Hospital Fund report the comments of Dr. R. E. Irvine (20 February, p. 524) are reasonable. It is striking, however, that nearly all those working with the elderly in Lewisham with whom I have discussed the relevant hospital services express the opinions that such services leave much to be desired, and that the report gives an incorrect representation.

How has this contradiction happened ? Firstly, the sample taken could hardly give an accurate assessment of the number of penple at home who should be in hospital. Taking the figures for the Deptford area, only four or five people would have been expected to have been found in the sample; that they were not does not necessarily mean that in the rest of the population there were none who should be in hospital. (This is quite a different order of significance from that of say, the home help service where $9 \%$ of the whole sample was involved.)

Secondly, the investigation relied upon the voluntary reporting of cases by general practitioners. This is a notoriously unreliable method, and a negative result is of no value.

Thirdly, the committee contained no consultant physician in geriatrics, and this is surely a grave omission when reporting on hospital services for the elderly. It is inconceivable that a paediatric survey would be conducted without a paediatrician, as only he is adequately qualified to judge in his special field. This is no idle quibble, as a committee will cnly get the right answers if it asks the right questions, and specialized knowledge is needed for these. For example, a high incidence of incontinence and bedfastness was discovered, but no attempt was made to ascertain how many cases were treatable, or could have been prevented in the first place.

Finally, the majority of the committee has direct connexions with the Lewisham Hospital Group. Is this a good way to obtain an impartial report?

I pose these criticisms because they may give clues as to how the committee returned a report at such variance with many people's opinions on the Lewisham hospital services for the elderly. Contrary to Dr. Meyrick's suggestion that no further inquiry is necessary, I feel it is urgently indicated.-I am, etc.,

$$
\begin{aligned}
& \text { St. Alfege's Hospital, } \\
& \text { London S.E.10. }
\end{aligned}
$$

on it because, although the meaning is clear to those acquainted with the subject, it could lead the less erudite into error.

An unexpected response to a drug is nowadays often described as a "sensitivity reaction." It is usually tacitly accepted that this means no more than that the occurrence of the response is unpredictable and inexplicable in the current state of knowledge, and does not necessarily imply sensitization as normally understood. It is thus unfortunate that the recommended procedure, which he described as a sensitivity test, superficially resembles some type of desensitization. When he goes on to assert that if a certain dose of analgesic causes no abnormal response these drugs can be used freely, he gives the impression that it is known that the response is idiosyncratic and independent of the relative doses of the interacting drugs. This may not be true. A patient who reacted normally to pethidine on one occasion, reacted abnormally on a second occasion when the dose of monoamine-oxidase inhibitors had been increased. $^{1}$ The patient he describes who responded abnormally to $5 \mathrm{mg}$. of pethidine might have reacted catastrophically to 100 mg., and it is because of this likelihood that a very small initial dose is advised.

Of more practical importance, however, is the impression he gives that analgesics are interchangeable in this context. All the

\section{Pelvic Endometriosis with Irreducible Femoral Hernia}

SIR,-Inguinal endometriosis, and endometriosis occurring in the walls of hernial sacs, have been described on rare occasions. Moore ${ }^{1}$ described a case of extensive pelvic endometriosis associated with bilateral inguinal herniae. Dreishpoon ${ }^{2}$ described two cases of endometrial deposits in the walls of hernial sacs-one inguinal and the other femoral-but in neither case was laparotomy done. Bornstein ${ }^{3}$ reports one case of endometriosis occurring in an umbilical hernia sac but unassociated with any uterine pathology. Jimenez ${ }^{4}$, Niswander, ${ }^{5}$ and Dormandy ${ }^{6}$ have all reported cases of inguinal endometriosis.

The following case illustrates an unusual finding during the course of femoral herniorrhaphy, in which the contents of the sac led to the discovery of extensive pelvic endometriosis which had hitherto remained undetected.

Mrs. A. B., aged 42 years, was referred to hospital because of a painful, tender swelling in the right groin of one day's duration. There was no previous history of hernia, or of the patient's "sensitivity." reports of this complication that $I$ have been able to trace seem to have followed the use of pethidine. One patient who died after pethidine had had no ill-effects after a previous dose of morphine. ${ }^{2}$ The establishment of a normal response to one analgesic does not, therefore, preclude a potentially fatal result with a different one. One would, therefore, like to qualify Dr. ChurchillDavidson's advice. One should investigate the response to the analgesic that it is intended to use, and then use no other. If time is short morphine is preferable to pethidine. The procedure is an attempt to establish whether or not a therapeutically adequate dose of the drug can be given without disastrous side-effects and does not modify

It is perhaps worth noting that the demonstration of a normal response to pethidine does not eliminate the possibility of a dangerous or fatal response to vasopressors, and this likelihood constitutes a valid reason to stop the monoamine-oxidase inhibitors as soon as possible during a time of potentially acute illness.-I am, etc.,

\section{Department of Anaesthetics, The University, Newcastle upon Tyne. REFERENCES : Taylor, D. C., Lancet, 1962, 2, 401.}

abdominal or gynaecological disease. Examination showed that she had an irreducible, tender right femoral hernia, but there was no clinical evidence of intestinal obstruction. She had one child following a normal delivery twenty years previously.

Femoral herniorrhaphy was undertaken later that day. Through a Lotheissen approach a large tense femoral hernia sac was found and opened. The sac was filled with altered blood but no bowel was evident. As many loops of bowel as could be delivered through the neck of the sac were examined and found to be normal. It was thought that the amount and nature of the sac content must indicate that a loop of bowel had strangulated, but that it may have returned to the abdominal cavity during manipulation of the hernia sac. The sac was excised and herniorrhaphy was performed. After closure of this incision the abdomen was opened through a right paramedian incision. The whole peritoneal cavity was extensively studded with altered blood, and a detailed examination of the intestine showed no abnormality or evidence of strangulation or incarceration. There was gross haemorrhagic staining over the uterus with "chocolate" cysts over both ovaries. The left

\section{Anaesthesia and Monoamine-oxidase Inhibitors}

SIR,-One welcomes the intention behind Dr. H. C. Churchill-Davidson's letter (20 February, p. 520). During the intervening weeks I have been hoping that a more authoritative pen than mine would comment

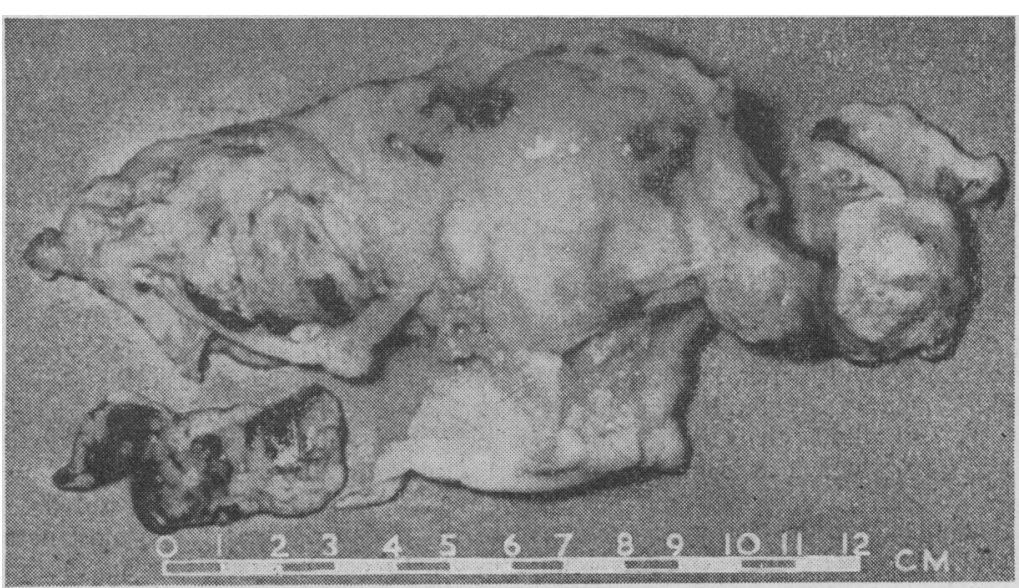

